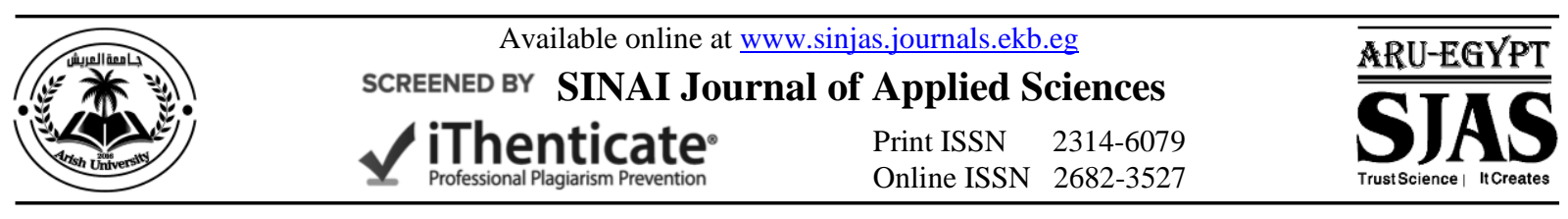

\title{
BIO-ECONOMIC STUDY OF AQUACULTURE INTEGRATED FISH TILAPIA (Oreochromis niloticus) UNDER THE DESERT ENVIRONMENT CONDITIONS (CASE STUDY), NORTH SINAI, EGYPT
}

\author{
Hamid M. Hamid ${ }^{1}$, M.S. Ahmed ${ }^{2}$ and M.A. Elshahed ${ }^{3}$ \\ 1. Environ. Studies Inst., Arish Univ., Egypt. \\ 2. Fac. Aquacul. and Fisheries Marine, Arish Univ., Egypt. \\ 3. Fac. Fisheries Sci., Suez Univ., Egypt.
}

\begin{tabular}{l} 
ARTICLE INFO \\
\hline Article history: \\
Received: 07/05/2021 \\
Revised: $25 / 05 / 2021$ \\
Accepted: 04/07/2021 \\
Available online: 04/07/2021 \\
\hline Keywords: \\
Bir al-Abd, \\
Nile tilapi, \\
aquaculture integrated, \\
Egypt \\
\\
Check for \\
updates
\end{tabular}

\begin{abstract}
This study was carried out in the year 2020, and the study area is located within the city of Bir al-Abd in the Ard al-Khair cluster in the Iqtiyah area, 15 $\mathrm{km}$ from the main road, al-Qantara al-Arish. The nature of the soil is sandy, and drip irrigation is on a daily basis. The predominant agricultural system is small farms. The main crops in the region are vegetable crops, and Nile tilapia was chosen as it is one of the most important freshwater fish as it is one of the most successful fish in small-scale farming as it achieves suitable and marketable market sizes. Therefore, the importance of the study comes through most of it the use of water and the production of fish in arid regions and the possibility Using them to irrigate plants, and take advantage of the nutrients they contain, while increasing the productivity of small farms without high technologies through the integration between agriculture and aquaculture. This is a pioneering idea in the field of mostly use and reducing waste in water while creating a kind of integration between the two parts of agricultural production, plant and animal. The results were recommending the use of water discharged from intensive fish breeding ponds in irrigation of vegetable farms because it provides the necessary fertilizers for plant growth and leads to increased productivity and income. It is also included in organic agriculture.
\end{abstract}

\section{مقدمة}

إن الاستزراع المائي الداخلي أحد أهم وأسرع ألإني

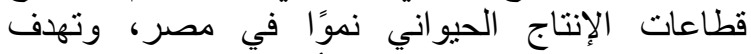

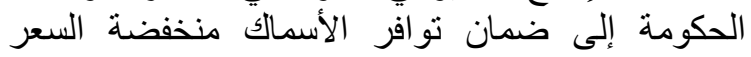

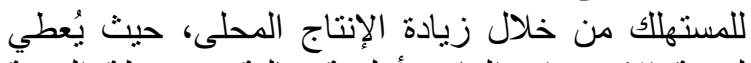

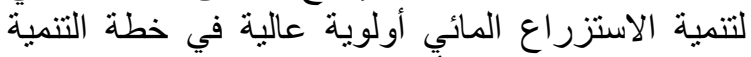

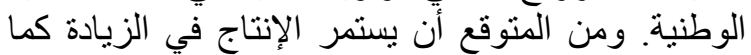

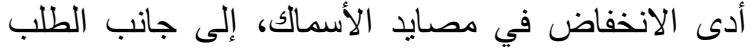

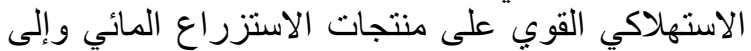

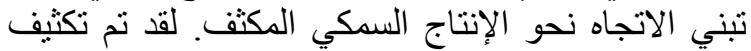

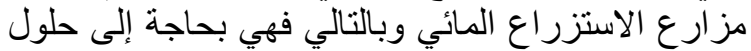

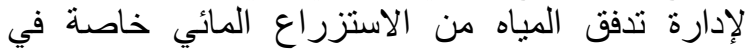

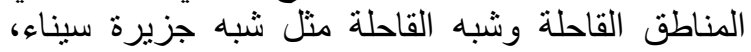

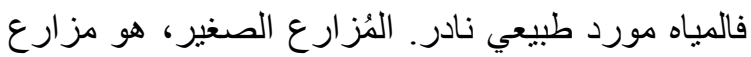

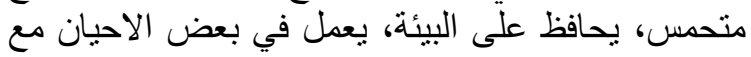

\footnotetext{
* Corresponding author: E-mail address: mansourhamidmansour@gmail.com https://doi.org/10.21608/sinjas.2021.75622.1021

(C) 2021 SINAI Journal of Applied Sciences. Published by Fac. Environ. Agric. Sci., Arish Univ. All rights reserved.
} 
المناطق الرطبة و الغزيرة، كما أن الاستزر اع المائي في

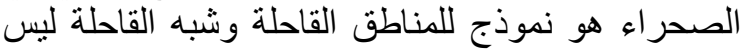
فقط في مصر ، ولكن أيضًا في البلدان الأفريقية الأخرى

.(Suloma and Ogata, 2006)

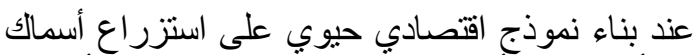

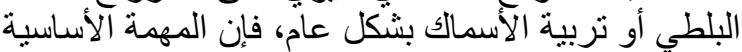

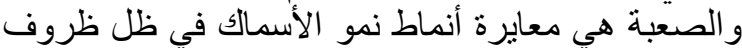

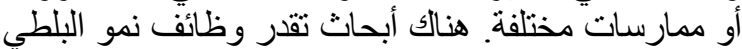
بناءً على البيانات التجريبية، و وعادة ما تحاكي هذه التهات البحوث

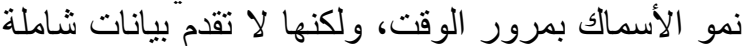

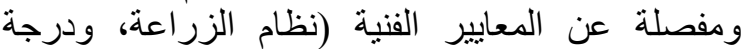

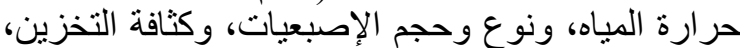

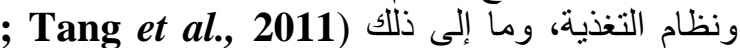

.(Santos, et al., 2013; FAO, 2018;

تفيد الدراسات البيو اقتصاديه في مساعدة المنتجين

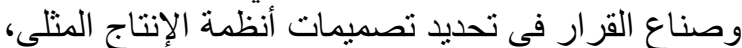
واستر اتيجيات الإدارة والخطط البديلة ( Pomeroy et

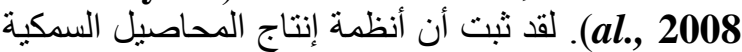
المتكاملة لها فو ائد اقتصادية وبيئية أكبر من أنظمة الإنتاج النياج

الأحادي (Da et al., 2015).

لذا تأتي اهمية الدراسة من خلال معظمه استخدام

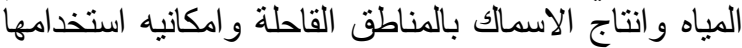

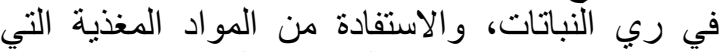

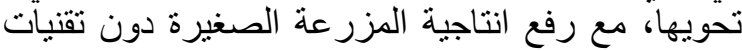
عالية عن طريق التكامل بين الزراعة والاعن الاستزراع المائي.

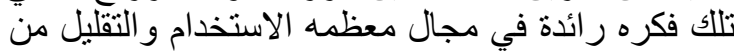

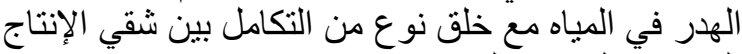
الزراعي النباتي والحيو اني.

\section{الطرق والأدوات}

لقد تمت هذه الدراسة في عام 2020 وتقع منطقة القائة

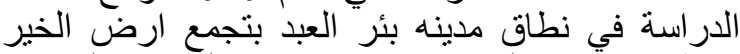

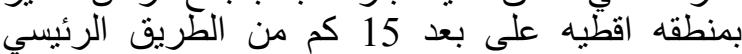
القنطرة العريش. طبيعة التربة رملية والرئي الرئ بالتنقيط

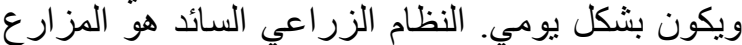

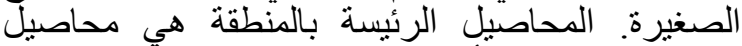
الخضر وتم اختبار أسماك البلطي النيلي (شكُل 1) حيث الثيث

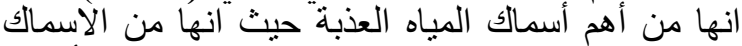

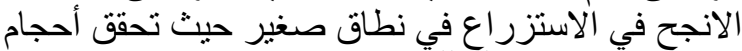
تسويقيه مناسبة و قابله للتسويق.

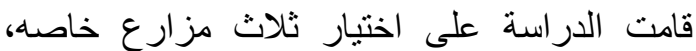
لتطبيق تربية الاسماك بالتكامل مع النبات تحت التر الظروف

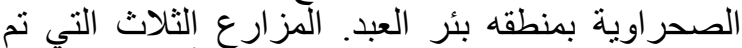

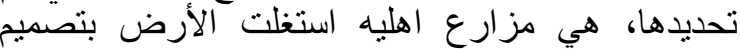

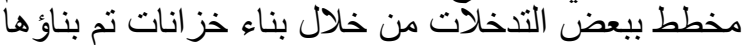
لتخزين مياه الري لزراعة النباتات. تضمنت النيات المزارع

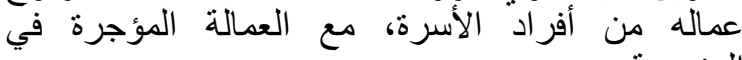

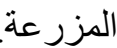

الاستزراع السمكي يعتبر اقتصادي غير مكلف ويحتاج

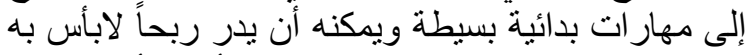

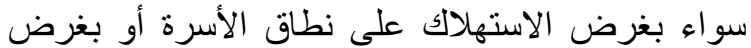

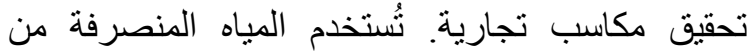

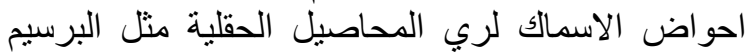

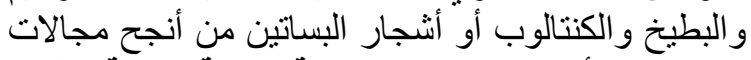

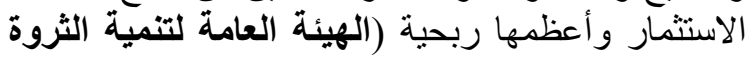

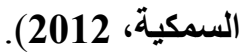

تُعد اسماك البلطي النبلي من أهم أسماك المياه العذبة فئة

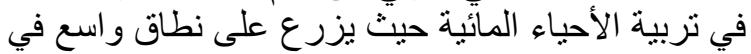
العديد من البلدان الاستوائية وشبه الاستو ائية في العالم في لئية (Coimbra and Reis-Henriques, 2005 and .Lin et al., 2008)

تم تسليط الضوء على الممارسات الحالية للاستزراع

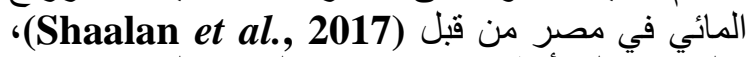

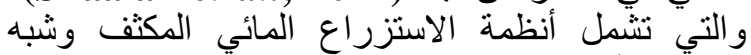

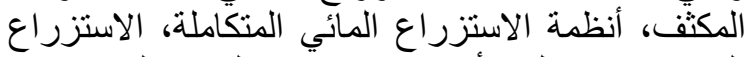

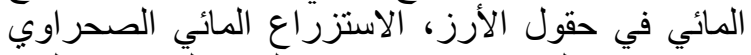

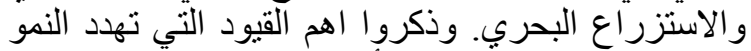

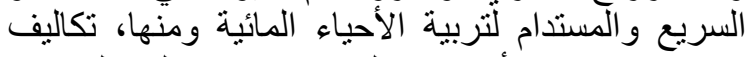

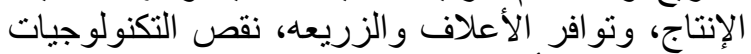
الحالية لإنتاج الأعلاف و اللو ائح المحلية.

البلطي النيلي، Oreochromis niloticus، أكثر

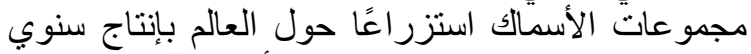

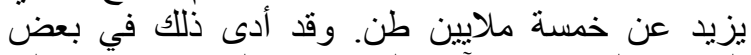

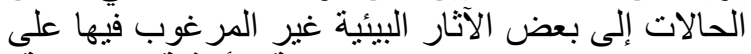
المستويين المحلي و العالمي (منظمة الأغذية والزئية الزراعة،

2019 و2020).

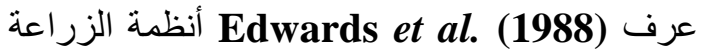

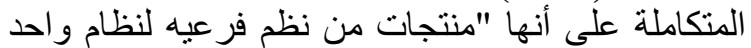

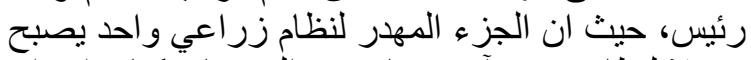

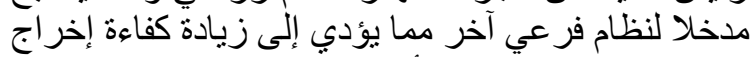
المنتجات المر غوبة من الأرض/ آلمياه.

أنظمة الأسماك المتكاملة مع المحاصيل، تشمل الأرباء

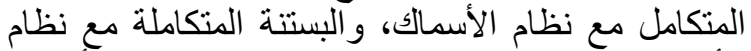
الأسماك، وتربية دودة القز المتكاملة مع نظام الأسماك الكاك والفطر المتكامل مع نظام الأسماك دودة الفكاكة Castro et al., 2006; Suloma and Ogata, 2006; Li and Gowing, 2008; Roman et al., .(2010; Martin et al., 2012

الزراعة المتكاملة من التوقعات المستقبلية الواعدة

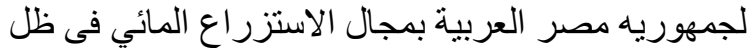
ندره المياه واهتمام الدولة بهذا القطاع (المنظمة العزية العربية للتنمية الزراعية، 2008) وتعتبر الزراه الزاعة المتكاملة بين

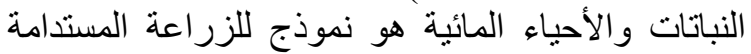

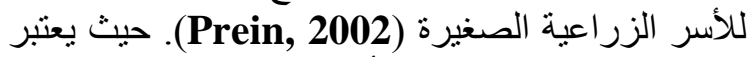
الاستزر اع السمكي نموذجًا لأساليب الاستزر اعناع المائي في بني 


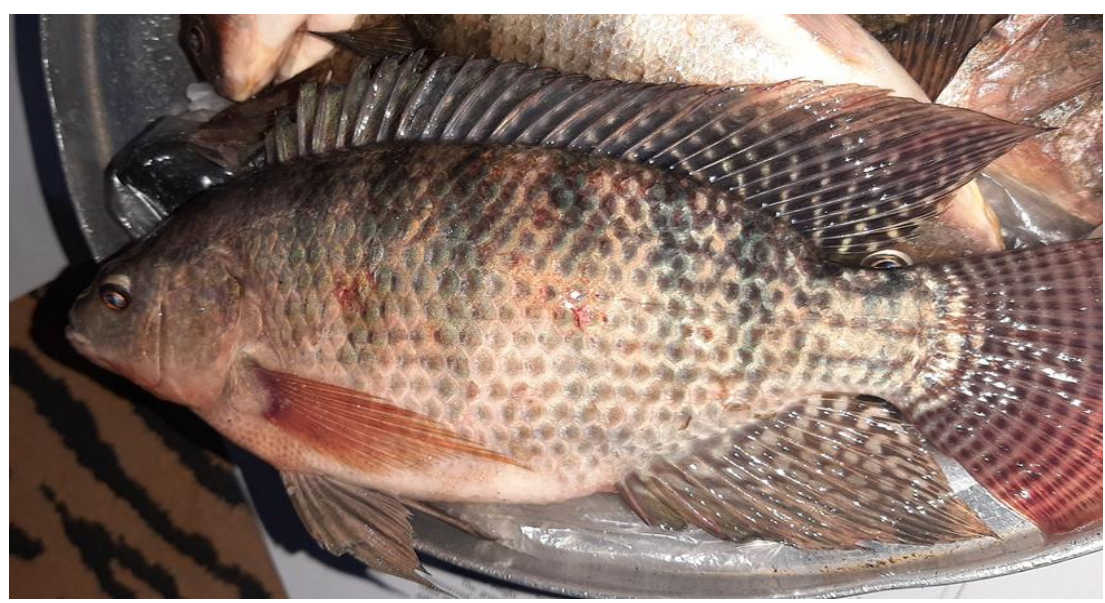

Oكل 1. سمكه البلطى Oreochromis niloticus

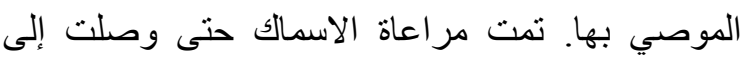

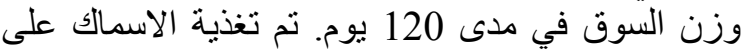
اعلاف مركزه (30 \% م فرونين).

\section{النتائج ومناقشتها}

في نهاية التجربة بعد 105 يوم، تم عرض نتائج تحليل الاوزان في جنول 2. متوسط الوزن النهائي للوحدة من اسمالك البلطي النيلي Oreochromis niloticus كاني 192.3 جم، 188 جم، 164.5 جم و 133.3 جم 138 جم على 15.3 التو الي، لمستويات تخزينيه مختلفة 10، 10 ، 15، 20 و 25

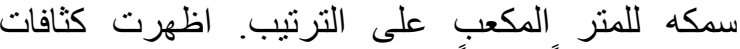

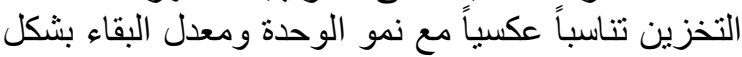

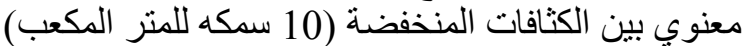
و المتوسطة (15 و20 سمكه للمتر المكعب) و العالية (25 (المنه

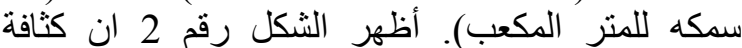

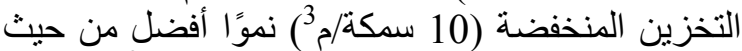

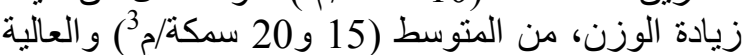

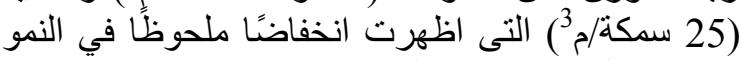
في نهاية الفترة عن بقية المعاملات.

بشير جدول 1 للممارسات الزراعية وإدماجها في زراعة ونربية الأحياء المائية في منطقة الدراسة لاستة بيئر

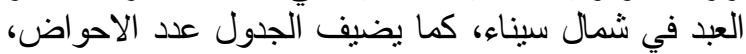

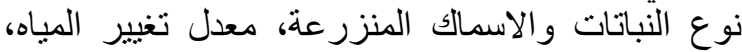

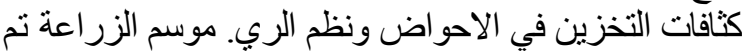

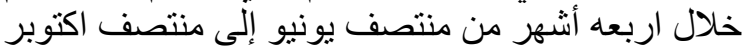

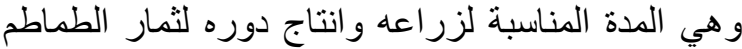
و هي النوع الاكثر زر اعه بالمنطقة.

كما تبين من الدراسة وجود فرق كبير في متوسط

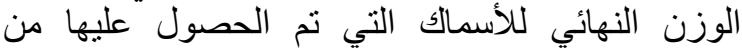

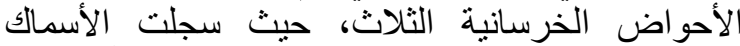
المأخوذة من النظام رقم 1 (الحوض الاكبر حجماً 390 $.1 .3 \times 25 \times 12$

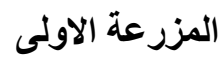

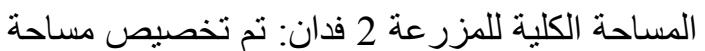

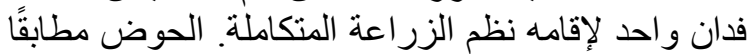

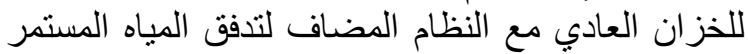

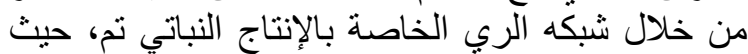
نزود الحوض بمصارف جيدة. تم تخزين الاسماك بكثافة بالإنة عالية (25 سمكه للمتر المكعب). محصول النوف الطماطم هو النوع النباتي المنزرع.

المزرعة الثانية

المساحة الكلية للمزر عة فدان واحد فقط: تم تخصيص

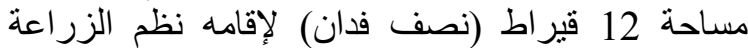
المتكاملة. تم تخزين الاسماك بكثافة متوسطة (20 سمكه

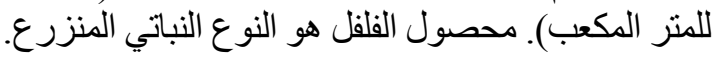

\section{المزرعة الثالثة}

المساحة الكلية للمزر عة 12 قير اط (نصف فدان) فقط. تم تخزين الاسماك بكثافة منخفضها (10 سمكه للمتر

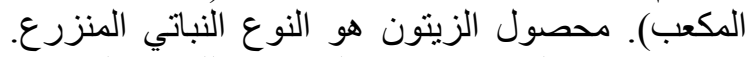

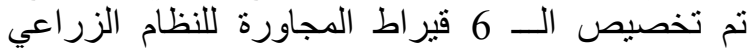
التقليدي بالمنطقة مع نفس نوع المحصول.

تم جمع البيانات، اعتمدت بشكل رئيسي على القياسات

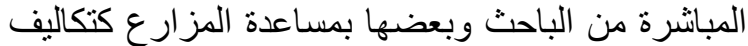
الانتاج و العائدات. بدأت الدرة استة على مستوى المجنمع

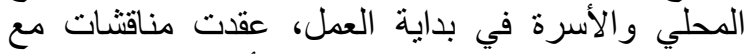

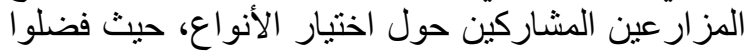

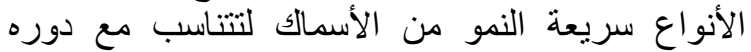

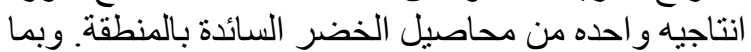

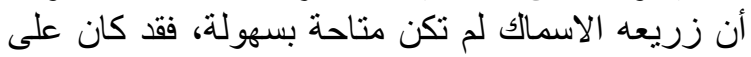

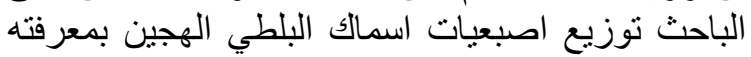

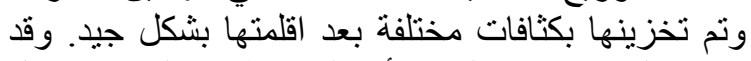

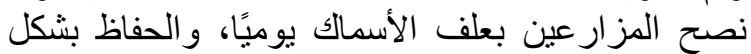
دوري على تغيير المياه والحفاظ على كثافة التخزين 


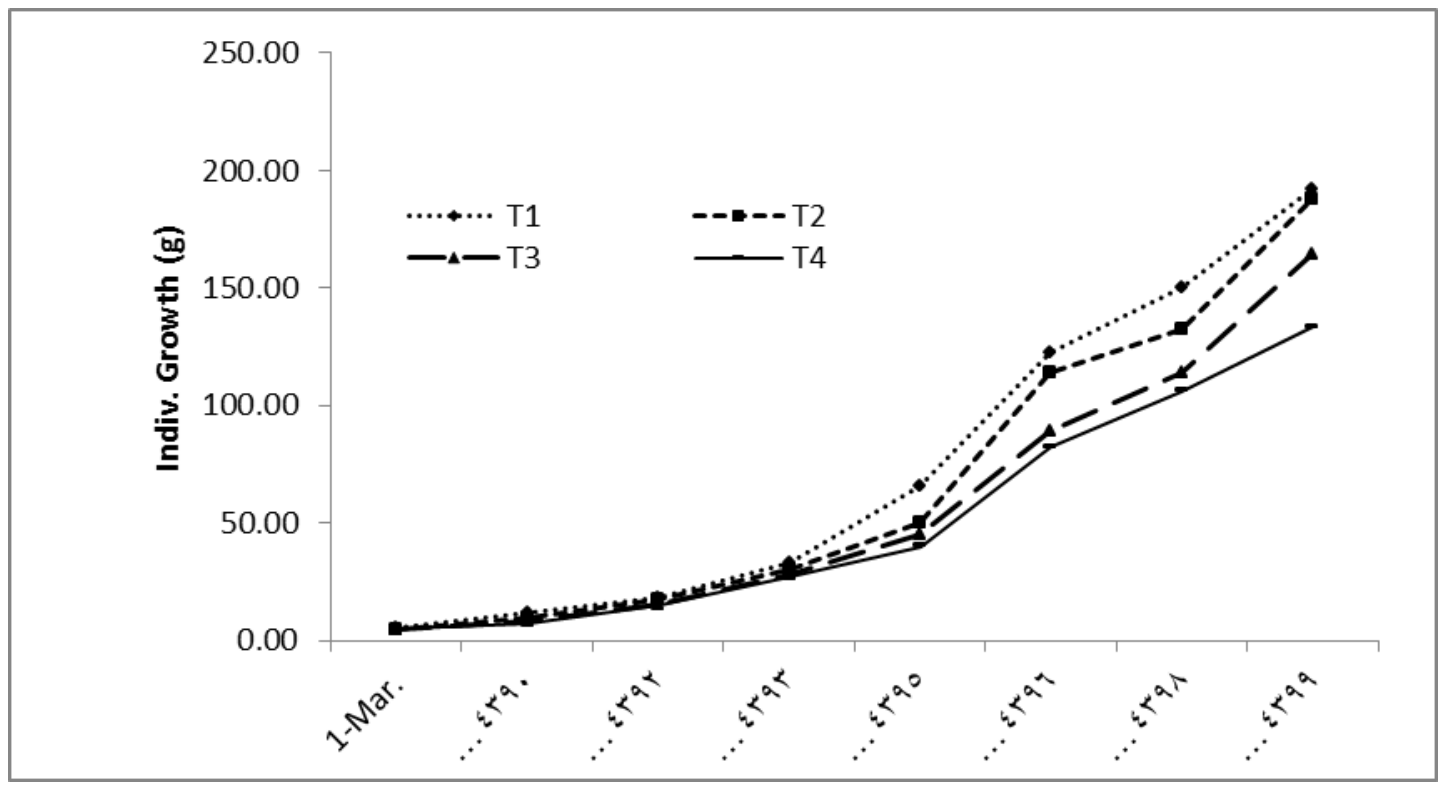

شكل 2. زيادة وزن الوحدة بمرور الوقت بكثافات تخزينه مختلفة

جدول 1. الممارسات الزراعية وإدماجها في زراعة وتربية الأحياء المائية في منطقة الدراسة

\begin{tabular}{llll}
\hline Farm and pond fish number & $\mathbf{1}$ & $\mathbf{2}$ & $\mathbf{3}$ \\
\hline Total farm size $\mathrm{m}^{3}$ & 4200 & 2100 & 900 \\
Water source for fish pond & Groundwater & Groundwater & Groundwater \\
Type of fish culture systems & Intensive & Intensive & Intensive \\
Species & Tilapia & Tilapia & Tilapia \\
Pond structures & concrete & concrete & Concrete \\
Aeration & Natural & Natural & Natural \\
Water exchange in $24 \mathrm{Hrs}$ & Full exchange & $50 \%$ Exchange & $25 \%$ exchange \\
Pond measurements & $12 * 25 * 1.3$ & $10 * 20 * 1.3$ & $4 * 5 * 1$ \\
Total water volume $\mathrm{m} 3$ & 390 & 260 & 20 \\
Fish density fish/m3 & 20 & 20 & 15 \\
Total fish & 7800 & 5200 & 300 \\
Planets & Tomato & Peppers & Olive trees \\
Irrigation system & Dropping & Dropping & Dropping \\
\hline
\end{tabular}




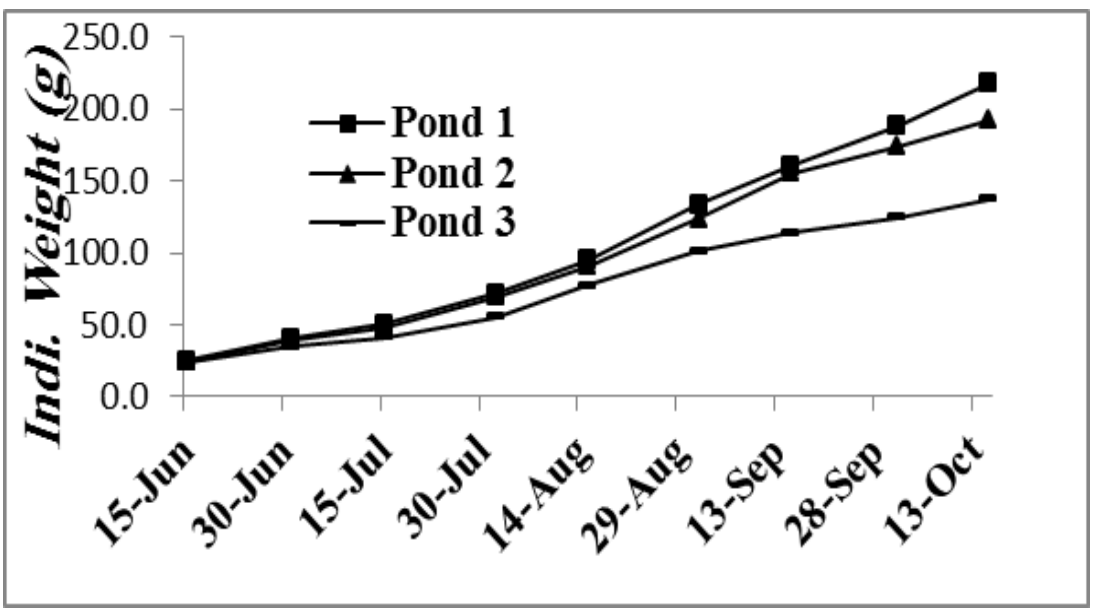

شكل 2. زيادة وزن الوحدة بمرور الوقت للأحواض

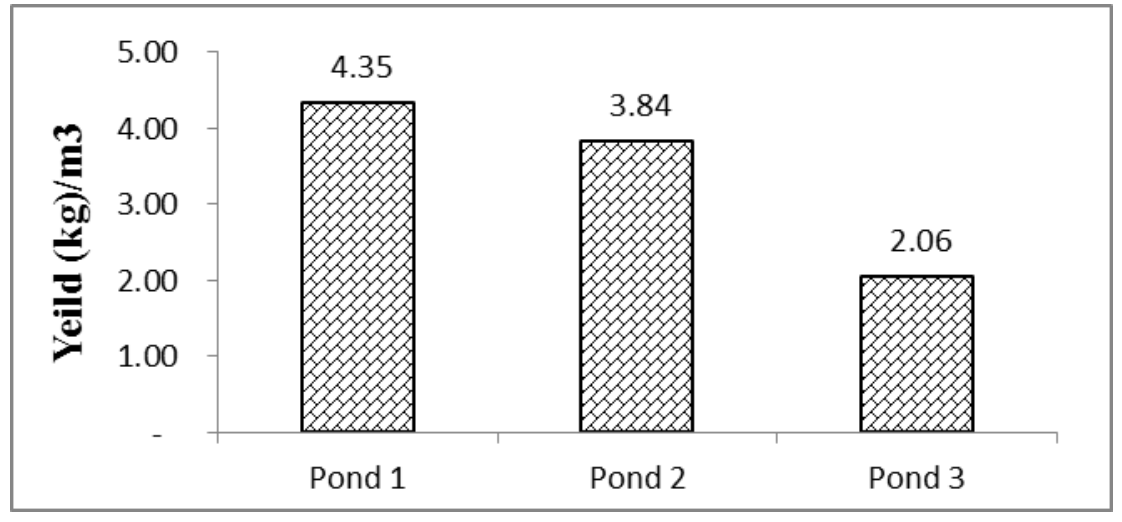

شكل 3. محصول الأسماك (كجم) لكل متر مكعب من المياه

نمو اً منماثلاً. كان معامل الانحدار الذي تم الحصول عليه

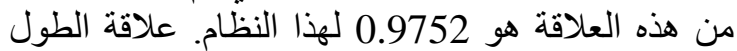

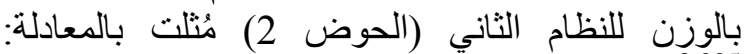

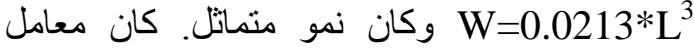

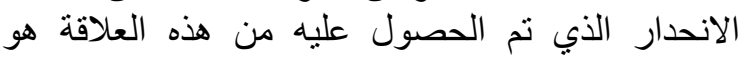

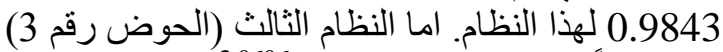
فأعطى نمواً منماتل ايضا بالمعادلة:

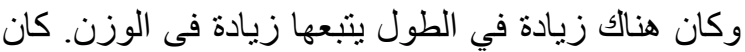

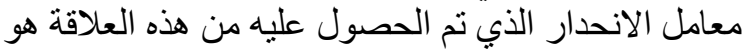

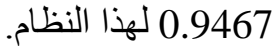

سجل معامل الحالة الجسمي 2.2 و و2.2 و 2.3 على الزي التوالي للنظم الزراعية الاول و الثاني و الثالث على على

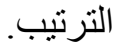

ومعدل تغيير المياه اليومي100\%) أعلى منوسط

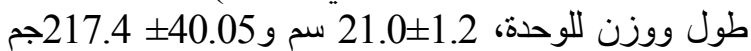

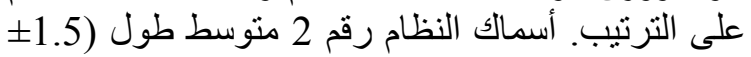

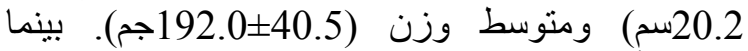
سجلت أسماك النظام رقم 3 اقل منوسط طول (1.5.5 (1.5.

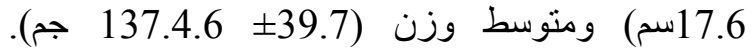
منوسط النمو للوحدة خلال فتره الدراسة واشتار لهذا شكل

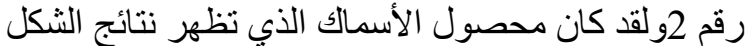

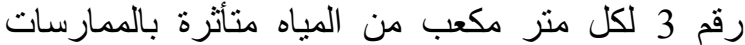

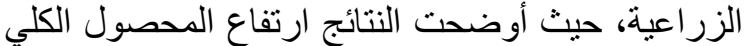

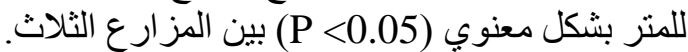
أوضح شكل 4 علاقة الطول بالوزن في اسماك

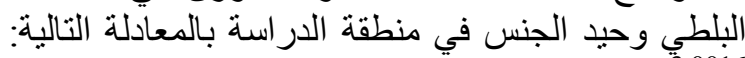
W=0.022*L $\mathrm{L}^{3.0016}$ 


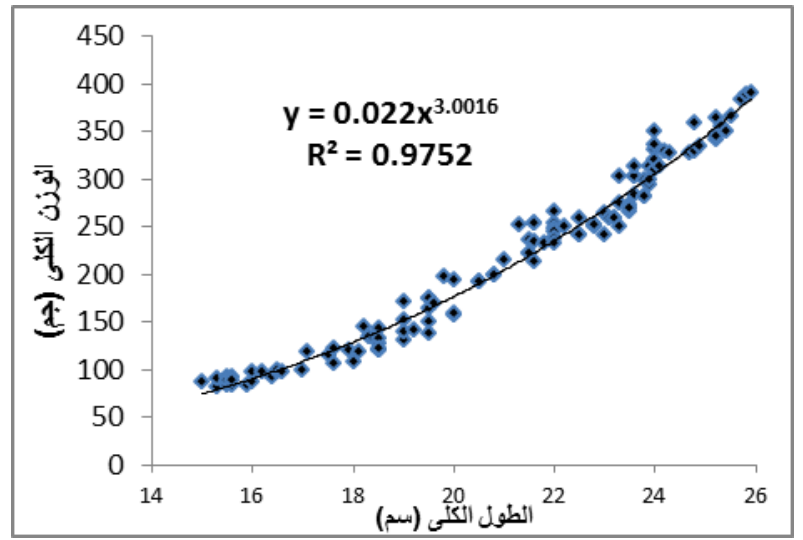

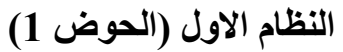

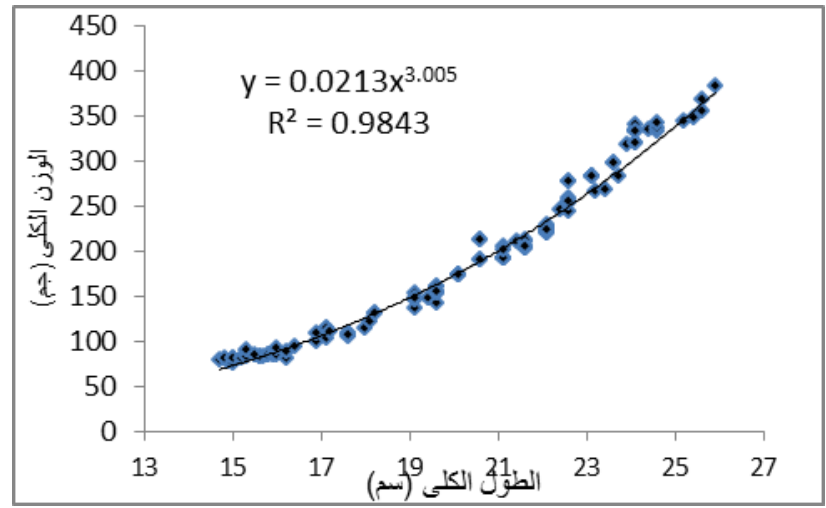

النظام الثاني (الحوض (2) 2)

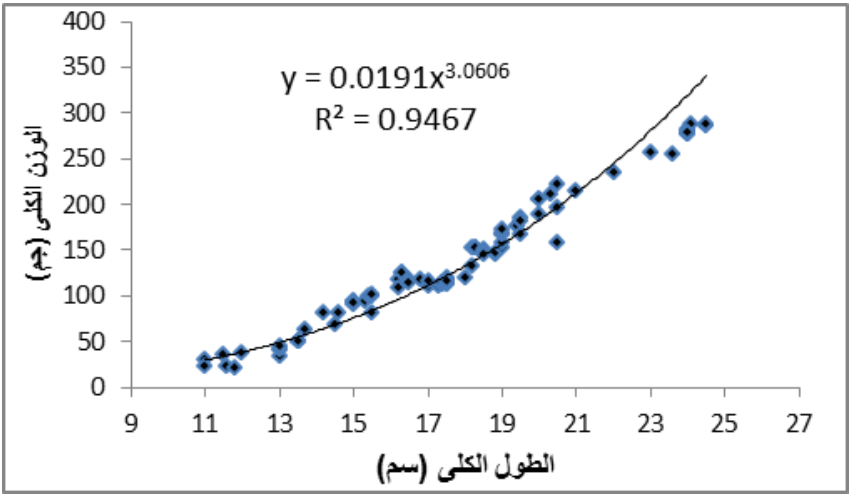

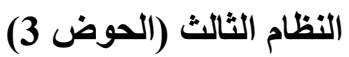

بينما بلغ إجمالي التكاليف لمزرعة الطماطم التقليدية

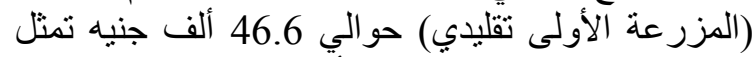

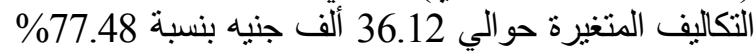
من إجمالي التكاليف وقدرت التكاليف الثابتة بحوالية التئية 10.5 ألف جنيه تمثل حوالي 22.5\% من إجمالي التكاليف

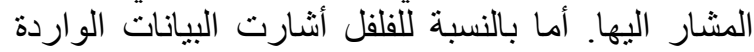

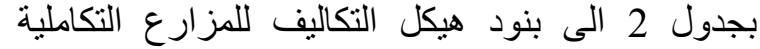

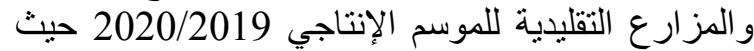

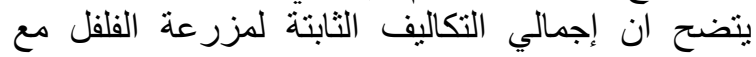

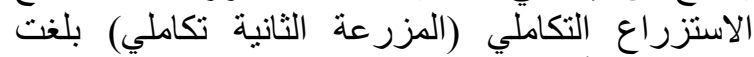

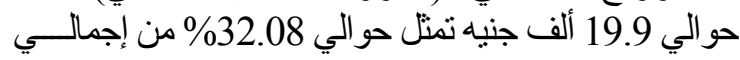

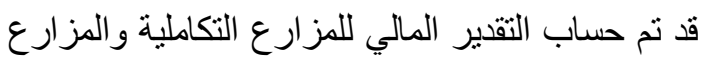

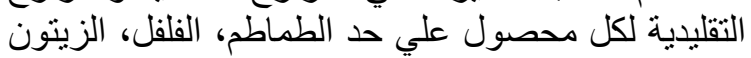

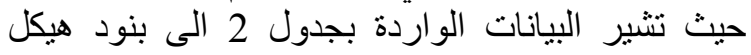

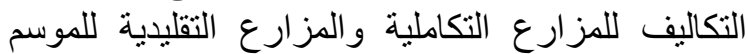

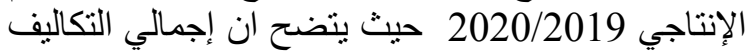

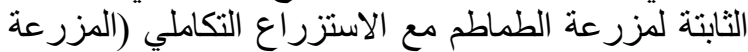

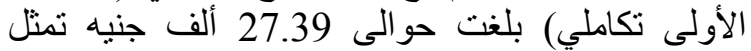
حو الى 26.67\% من إجمالي التكاليف الكلية بينما قدرت التكاليف المتغيرة بنحو 75.33 ألف جنيه تمثل حوالى الى لئل

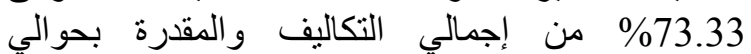
102.713 ألف جنيه. 
جدول 2. بنود هيكل التكاليف لمزارع العينة لمحاصيل (الطماطم_القلقل_الزيتون) خلال الموسم الإنتاجي 2020/2019

\begin{tabular}{|c|c|c|c|c|c|c|c|c|c|c|c|c|}
\hline \multirow{2}{*}{\multicolumn{2}{|c|}{ المزرعة الثالثة }} & \multicolumn{4}{|c|}{ مزارع تقليدية } & \multicolumn{6}{|c|}{ مزارع تكاملية } & \multirow{3}{*}{ ألتكاليف الثابتة } \\
\hline & & \multicolumn{2}{|c|}{ المزرعة الثانية } & \multicolumn{2}{|c|}{ المزرعة الاولى } & \multicolumn{4}{|c|}{ المزرعة الثانية المزرعة الثالثة } & \multicolumn{2}{|c|}{ المزرعة الاولى } & \\
\hline$\%$ & قِيمة & $\%$ & قيمة & $\%$ & قيمة & $\%$ & قيمة & $\%$ & قيمة & $\%$ & قَيمة & \\
\hline 92.6 & 5000 & 30 & 3000 & 13.3 & 3000 & 75.6 & 8075 & 66.2 & 14500 & 49 & 19290 & احواض+ بئر \\
\hline 9.3 & 500 & 3 & 300 & 1.3 & 300 & 7.6 & 808 & 6.6 & 1450 & 4.9 & 1.929 & اهلاك اننشاءات \\
\hline 0 & 0 & 0 & 0 & 0 & 0 & 1.9 & 200 & 1.8 & 400 & 1.5 & 600 & مواسير رى وصرف \\
\hline 0 & 0 & 25 & 2500 & 23.8 & 2500 & 0 & 0 & 11.4 & 2500 & 6.3 & 2500 & موتور سحب \\
\hline 7.4 & 400 & 25 & 2500 & 47.6 & 5000 & 3.7 & 400 & 11.4 & 2500 & 12.7 & 5000 & شبكة رى نبات \\
\hline 7.4 & 400 & 50 & 5000 & 71.4 & 7500 & 5.6 & 600 & 24.7 & 5400 & 20.6 & 8100 & \\
\hline 0.7 & 40 & 6.25 & 500 & 4.14 & 750 & 0.6 & 60 & 2.5 & 540 & 2.1 & 810 & ا هلاك الات ومعدات \\
\hline 100 & 5400 & 80 & 8000 & 85 & 10500 & 81 & 8675 & 91 & 19900 & 70 & 27390 & اجمالى التكاليف الثابتة \\
\hline 0 & منزلى & 57 & 12000 & 33.2 & 12000 & 0 & 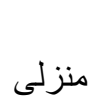 & 38 & 16000 & 21.24 & 16000 & عماله \\
\hline 60.9 & 800 & 9.5 & 2000 & 5.5 & 2000 & 51.9 & 400 & 4.7 & 2000 & 2.66 & 2000 & كهرباء \\
\hline 0 & 0 & 4.9 & 1040 & 13.8 & 5000 & 0 & 0 & 2.5 & 1040 & 6.64 & 5000 & ش شتلات \\
\hline 7.6 & 100 & 4.8 & 1000 & 22.2 & 8000 & 6.5 & 50 & 2.4 & 1000 & 10.62 & 8000 & مبيدات \\
\hline 15.2 & 200 & 14.3 & 3000 & 16.6 & 6000 & 13 & 100 & 7.1 & 3000 & 7.97 & 6000 & سماد \\
\hline 16.3 & 215 & 9.5 & 2000 & 8.7 & 3125 & 11.7 & 90 & 5 & 2100 & 5.67 & 4273 & صيانة \\
\hline 0 & 0 & 0 & 0 & 0 & 0 & 9.1 & 70 & 6.6 & 2800 & 6.97 & 5250 & اجمالى ثمن الزريعه \\
\hline 0 & 0 & 0 & 0 & 0 & 0 & 7.8 & 60 & 33.7 & 14200 & 38.23 & 28800 & تكاليف علف \\
\hline \multirow[t]{4}{*}{100} & 1315 & 100 & 21040 & 100 & 36125 & 100 & 770 & 100 & 42140 & 100 & 75323 & اجمالى التكاليف المتغيرة \\
\hline & 6715 & & 29040 & & 46625 & & 9445 & & 62040 & & 102713 & اجمالى التكاليف \\
\hline & 19.58 & & 72.45 & & 77.48 & & 8.15 & & 67.92 & & 73.33 & التكاليف المتغيرة \% \\
\hline & 80.42 & & 27.55 & & 22.52 & & 91.85 & & 32.08 & & 26.67 & التكاليف الثابتة \% \\
\hline
\end{tabular}

بينما بلغ إجمالي التكاليف للمزرعة الزيتون التقليدية

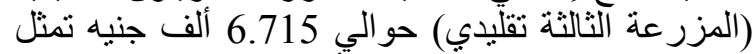
التكاليف المتغيرة حو الي 1.315 ألف جنيه بنسبة 19.58 من إجمالي التكاليف وقدرت التكاليف التئ الثابتة بحوالي التي 5.400 ألف جنيه تمثل حوالي 80.42\% من إنمالي الئي التكاليف المشار إليها.

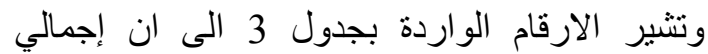
الإيرادات لمزرعة الطماطم التكاملية حوالي

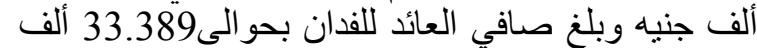
جنيه اما في المزرعة التقليدية بلغ إجمالي العائد النباتي لئي

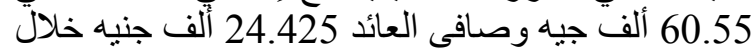
الموسم الإنتاجي 2020/2019.

كذلك بالنسبة لمزرعة الفلفل المتكاملة كان إجمالي

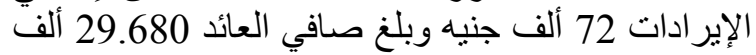

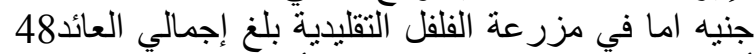

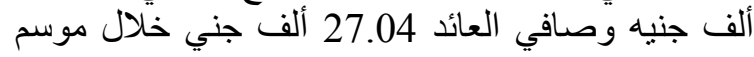

التكاليف الكلية بينما قدرت التكاليف المتغيرة بنحو

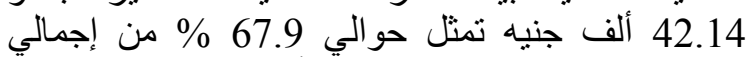
التكاليف و المقدرة بحو الي 62.04 ألف جنيه

بينما بلغ إجمالي التكاليف للمزرعة الفلفل التقليدية

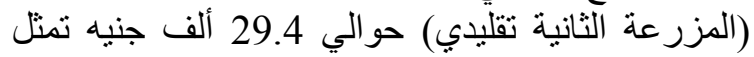

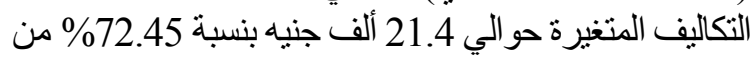

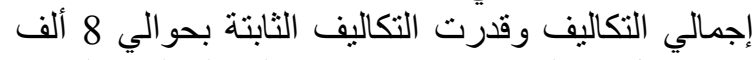
جنيه تمثل حوالى 27.55\%من إجمالي التكاليف المشار

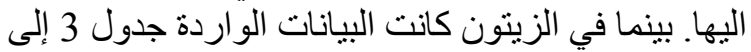

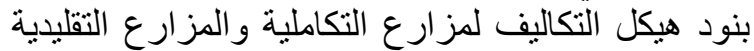

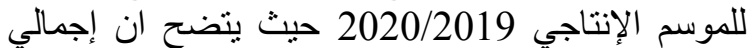

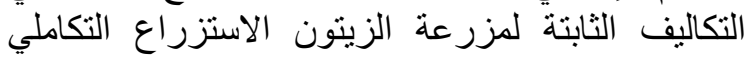

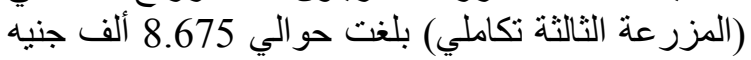
تمثل حو الي 91.85\% من إجمالي التكاليف الكلية بينما

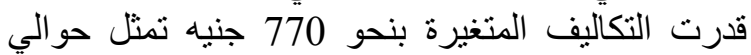
8.15\% من إجمالي التكاليف و المقدرة بحو الي 9.45 ألف تلفي 
جلول 3. كمية الانتاج وسعر المنتج وصافي العائد من المزارع التكاملية والمزارع التقليدية خلال الموسم الإتتاجي 2020/ 2019

\begin{tabular}{|c|c|c|c|c|c|c|}
\hline \multicolumn{3}{|c|}{ مزارع تقليلية } & \multicolumn{3}{|c|}{ مزارع تكاملية } & \multirow[t]{3}{*}{ العائد/مساحة } \\
\hline زيتون & فلفل & طماطم & زيتون & فلفل & طماطم & \\
\hline 400 & فدان & فـان & 2400 & فدان & فذان & \\
\hline 1000 & 9,600 & 17,300 & 1,200 & 10,000 & 20,400 & الانتاج /كجم \\
\hline 7 & 5 & 3.5 & 7 & 5 & 3.5 & متوسط السعر (جنيه) \\
\hline \multirow[t]{5}{*}{7,000} & 48,000 & 60,550 & 8,400 & 50,000 & 71,400 & إجمالي عائد نباتي (جنيه) \\
\hline & & & 41.2 & 1000 & 1696 & الانتاج /كجم \\
\hline & & & 15 & 22 & 22 & متوسط السعر (جنيه) \\
\hline & & & 0.62 & 22000 & 37312 & إجمالي عائد سمكي (جنيه) \\
\hline & & & 9,020 & 72000 & 108.712 & إجمالي عائد سمكي + نباتي \\
\hline 5,685 & 27.040 & 24,425 & 8.250 & 29,860 & 33.489 & صافى العائد (جنيه) \\
\hline
\end{tabular}

تكون الموارد مثل الأرض و المياه ور أس المال غير كافية

.(Kapinga et al., 2014)

كان هنالك فرق كبير في منوسط الوزن النهائي للأسماك التي تم الحصول عليها من الأحو اض اضن الخرسانية

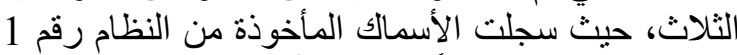

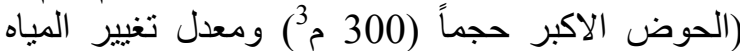

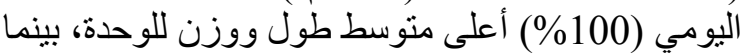
سجلت أسماك النظام رقم 3 اقل متوسط طول ومنوسط للوحة لونط

لقد أنشار Bagenal and Tesch, 1978 ان البيئة bكون ملائمة للنوع إذا كانت قيمة معامل الانحدار

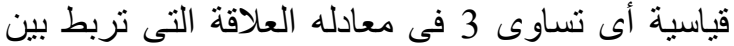

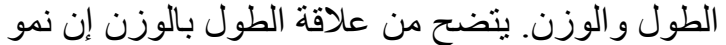

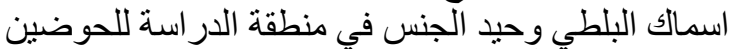

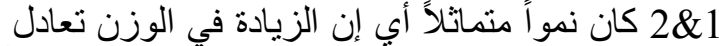

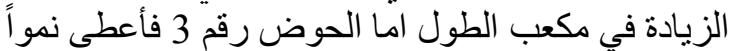

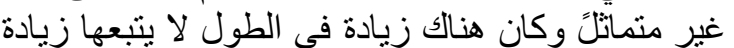

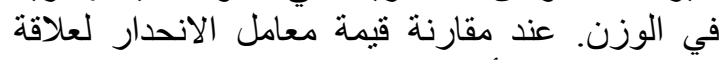

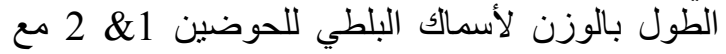

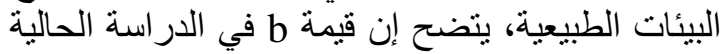

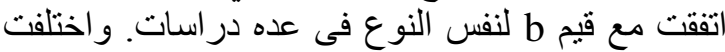

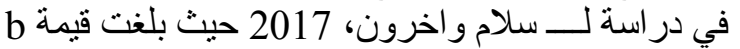
(2.01) لعلاقة الطول الكلي بالوزن الكلي وهذا يشير لئي إلى أن نمو سمكة البلطي النيلي ليس قياسيا في هذان الئل

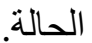

أيضا بالنسبة لمزرعة الزيتون المتكاملة بلغ إجمالي

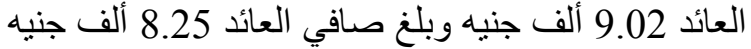

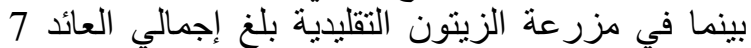
الاف جنيه وصافي العائد 5.685 ألف جنيه لئن الفيها

لقد تشابهات النتائج مع تللك التي حصل علديه

Chakraborty and Banerje (2010) وAlhassan et al. (2012) الذين لاحظوا انخفاض

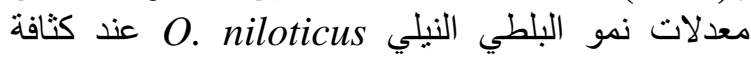

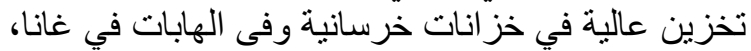
و المرباة في أحواض في سهول الغنانج في في غرب النيان البنغال،

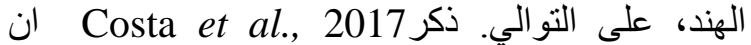
الزيادة في كثافة التخزين ادت إلى التخفاض التئ الوزئ الوزن النهائي

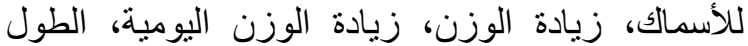

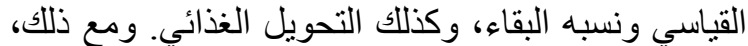
ذكر ان أن الكثافة العالية قد تقلل من التأثثر على التغير التيرات

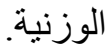

تبين فى التجربة ان النمو أعلى بشكل ملحوظ في

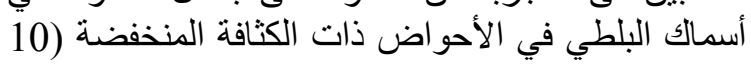

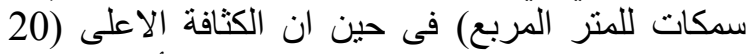

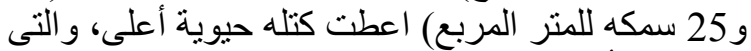

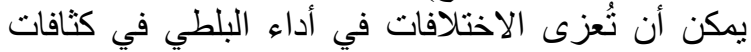
التخزين إلى الإجهاد و التنافس على الغذات الغذاء ومساحة المعيشة.

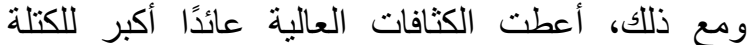

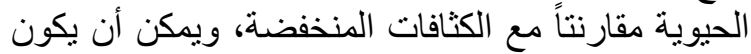
مفيدًا جدًا لتحقيق عو ائد جيدة للاستهلاك المحلي وحيث 
الهيئة العامة لتنمية الثروة السمكية (2012).

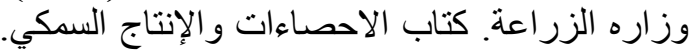
http://www.gafrd.org/ information.GAF

RD@gmail.com www.GAFRD.org

الهيئة العامة لتنمية الثروة السمكية (2017).

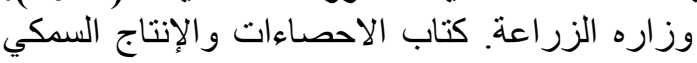
http://www.gafrd.org/ information.GAF

RD@gmail.com www.GAFRD.org,

Alhassan, E.H.; Abarike, E.D. and Ayisi, C.L. (2012). Effects of stocking density on the growth and survival of Oreochromis niloticus cultured in hapas in a concrete tank. Afr. J. Agric. Res., 7: 2405 - 2411.

Bardach, J.E.; Ryther, J.H. and McLarney, W.O. (1978). Aquaculture - the farming and husbandry of freshwater and marine organisms. John Wiley and Sons, INC, 351.

Bhatnagar, A. and Singh, G. (2010). Culture fisheries in village ponds: a multi-location study in Haryana, India. Agric. Biol. J. N. Ame., 1(5): 961-8.

Bhatnagar, A.; Jana, S.N.; Garg, S.K.; Patra, B.C.; Singh, G. and Barman, U.K. (2004). Water quality management in aquaculture. In: Course manual of summer school on development of sustainable aquaculture technology in fresh and saline waters, CCS Haryana Agric., Hisar (India), 203-210.

Bolger, T. and Connolly, P.L. (1989). The suitable of suitable indices for the measurement analysis of fish condition. J. Fish Biol., 34 (2): 171-182.

Boyd, C. (2004). Vesicles carry most exocyst subunits to exocytic sites marked by the remaining two subunits, $\operatorname{Sec} 3 \mathrm{p}$ and Exo70p. J. Cell. Biol., 167 (5): 889901 PMID :15583031.

Castro, R.S.; Azevedo, C.M.S.B. and Bezerra-Neto, F. (2006). Increasing cherry tomato yield using fish effluent as irrigation water in Northeast Brazil. Scientia Hort., 110.
معامل الحالة هي إحدى الممارسات المعيارية والتي

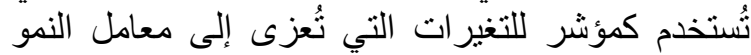

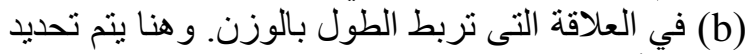

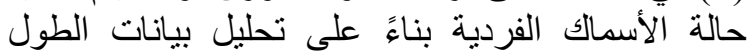
و الوزن و التي تعكس أن الأسماك الأثقل عند نفس الطول تكون في حالة أفضل (Bolger and Connolly, 1989). ذكر هاثثم وعبد المحسن، 2012، ان معامل الحالة

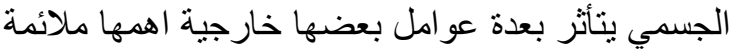
العو امل البيئية و الاخرى داخلية تتعلق بنشاط التغذية. بلغ معدل معامل الحال لسمكة البلطي النيلي خلال مدة

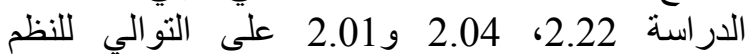
الزراعية الاول والثاني والثالث على الترتيب. معامل

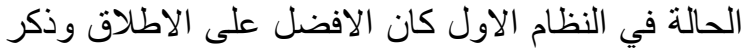
سلام واخرون، 2017 أن معامل الحالة الإلة الجسمي لأسماك الكالك

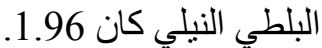

ربما يعود فرق النمو في المزارع الثلاث الى الى الثي المستويات المختلفة من الاكسجين المذاب نظر العيا لاختلاف

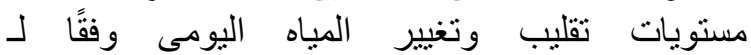
Bhatnagar و Bhatnagar and Singh (2010)

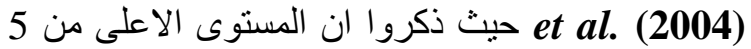

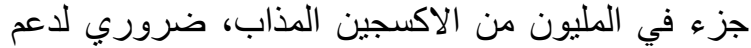

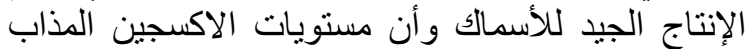

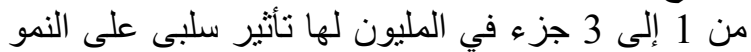

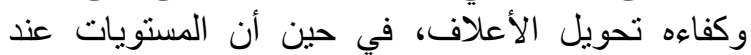

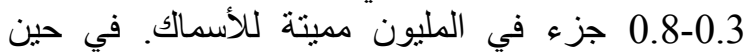

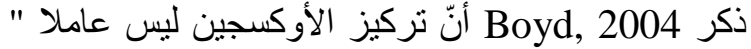

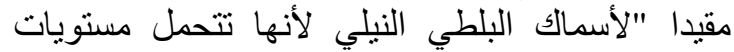
منخفضة تصل الى 3-4 ملغم /لتزر. التوصيات

لقد كانت نسبه الإعاشة كانت عالية والنمو جيد

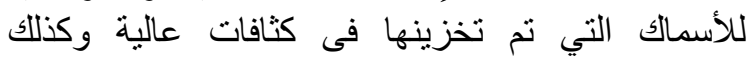
الأسماك التي تم تربيتها مع مدخلات عالية فئات (كثافات مرتفعة، حجم المياه والمعدل العالي لتغيير المياه المياه اليومي)

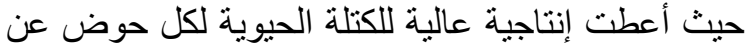
الأسماك المخزنة تحت مدخلات منخفضها لاعنه او كثنافات

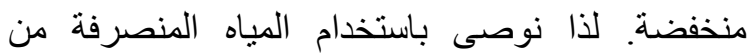

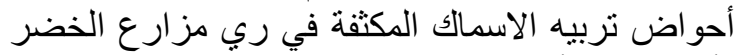

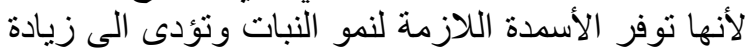

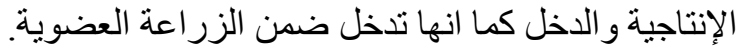

$$
\text { المراجع }
$$

المنظمة العربية للتنمية الزراعية (2008). دراسة حول

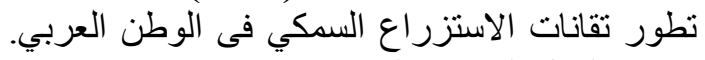
جامعه الدول العربية، الخرطوم. 
Li, Q.F. and Gowing, J.W. (2008). Investigation of integrated management of large-scale irrigation and aquaculture systems. J. Hydrol. Eng., 13: 355-363.

Lin, Y.H.; Lin, S.M. and Shiau, S.Y. (2008). Dietary manganese requirements of juvenile tilapia, Oreochromis nilotis $\mathrm{x}$ O.aureus. Aquac., 284: 207-210.

Martin, M.L.M.; Paez-Osuna, F.; Luis Esquer-Mendez, J.; Guerrero-Monroy, I.; Romo del Vivar, A. and FelixGastelum, R. (2012). Integrated culture of white shrimp (Litopenaeus vannamei) and tomato (Lycopersicon esculentum Mill) with low salinity groundwater: Manag. and production. Aquac., 366: 7684.

Mohamed, S.; El-Mahdy, M.; Theiner, S.; Dinhopi, N.; El-Matbouli, M. and Saleh, M. (2017). Silver nanoparticles: Their role as antibacterial agent against Aeromonas salmonicida subsp. salmonicida in rainbow trout (Oncorhynchus mykiss), Res. Vet. Sci. J. homepage: www. elsevier.com/locate/rvsc.

Pomeroy, R.; Bravo-Ureta, B.E.; Solís, D. and Johnston, R.J. (2008). Bio-economic modelling and salmon aquaculture: an overview of the literature. Int. J. Environ. Pollut., 33 (4): 485-450.

Prein, M. (2002). Integration of aquaculture into crops-animal systems in Asia. Agricultural system 71, 127-146.

Roman Poot-Lopez, G.; Hernandez, J.M. and Gasca-Leyva, E. (2010). Input management in integrated agricultureaquaculture systems in Yucatan: Tree spinach leaves as a dietary supplement in tilapia culture. Agric. Systems, 103: 98104.

Santos, V.B.; Mareco, E.A. and Silva, M.D.P. (2013). Growth curves of Nile tilapia (Oreochromis niloticus) strains cultivated at different temperature. Acta Scientiarum, 35: 235-242.
Chakraborty, B.S. and Banerjee, S. (2010). Effect of Stocking Density on Monosex Nile Tilapia Growth during Pond Culture in India. World Acade. Sci., Eng. and Technol. Int. J. Anim. and Vet. Sci., 4 (8): 646-650.

Coimbra, A.M. and Reis-Henriques, M.A. (2005). Nile tilapia, Oreochromis niloticus L., reproduction inhibition by dietary exposure to Aroclor 1254. Bulletin of Environ. Contamination and Toxicol., 75: 407-412.

Da, C.T.; Phuoc, L.H.; Duc, H.N.; Troell, M. and Berg, H. (2015). Use of wastewater from striped catfish (Pangasianodon hypophthalmus) pond culture for integrated rice-fish-vegetable farming systems in the Mekong Delta, Vietnam. Agroecol. Sustain. Food Syst. 39: 580-597.

Edwards, P.; Pullin, R.S.V. and Gartner, J.A. (1988). Research and education for the development of integrated croplivestock-fish farming systems in the tropics, ICLARM Studies and reviews 16, Int. Cent. Living Aquatic Res. Manag., Manila, Philippines, 139-170.

FAO (2001). Food and Agriculture Organization of The United Nations), Integrated agriculture-aquaculture: a primer. FAO Fisheries Techn. Rome, Italy, 149: 407.

FAO (2018). a. Improving the performance of tilapia farming under climate variation. Perspective from Bioeconomic Modeling. Rome, 2018.

Kapinga, I.; Enock, M. and Nasser, K. (2014). Effect of Stocking Density on the Growth Performance of Sex Reversed Male Nile Tilapia (Oreochromis niloticus) Under Pond Conditions in Tanzania. World J. Fish and Marine Sci., 6 (2): 156-161. 
Tang, Z.; Lin, Y.; Yang, H.; Zhang, Y.; Chen, Z.; Huang, Y.; Peng, T. and Zhang, Y. (2011). Growth model of GIFT strain tilapia (Oreochromis niloticus), Guangdong Agric. Sci., 18: 104-107.
Suloma, A. and Ogata, H.Y. (2006). Future of Rice-Fish Culture, Desert Aquaculture and Feed Development in Africa: The Case of Egypt as the Leading Country in Africa. JARQ, 40 (4): 351-360. 


$$
\text { الملخص العربي }
$$

دراسة بيو اقتصادي للاستزراع المائي المتكامل للأسماك البلطي النيلي (Oreochromis niloticus)

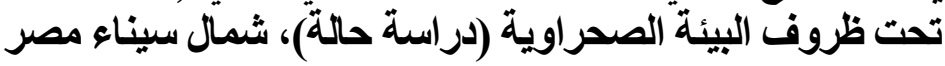

حميد منصور حميد1، محمد سالم أحمد²، محمد علي الثـاهد3

$$
\begin{aligned}
& \text { 1- - معهد الدراسات البيئية، جامعة العريش، مصر. }
\end{aligned}
$$

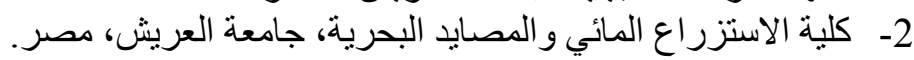

$$
\begin{aligned}
& \text { 3- كلية علوم الثروة السمكية، جامعة السويس، مصر . }
\end{aligned}
$$

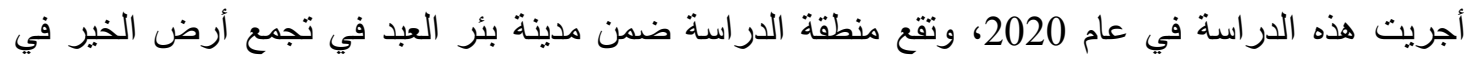

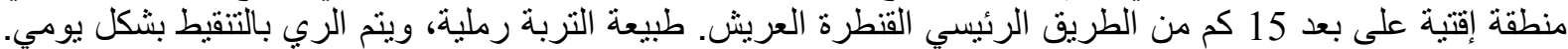

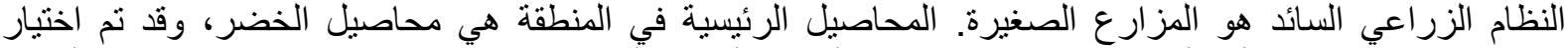

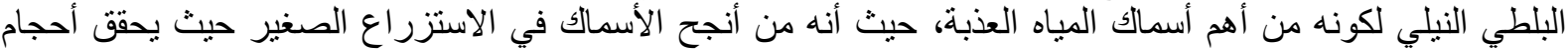

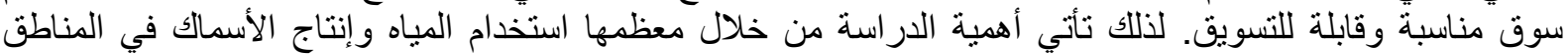

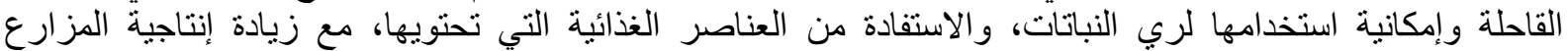

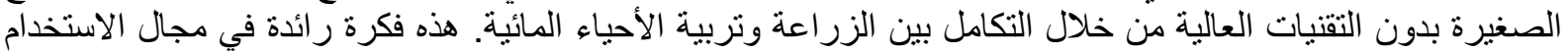

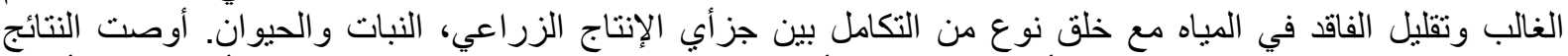

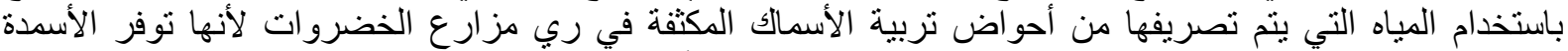

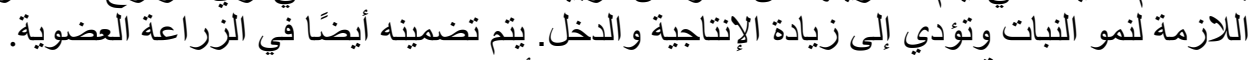
الكلمات الاسترشادية: بئر العبد، البلطي النيلي، نربية الأحياء المائية المتكاملة، مصر.

أستاذ الاقتصاد الزر اعي، كلية العلوم الزر اعية البيئية، جامعة العريش، مصر.

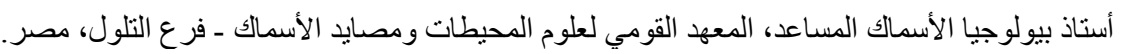

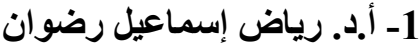
2- د. عطيه على عمر العيــاط أديان 